Case Report

\title{
Levofloxacin and moxifloxacin resistant Haemophilus influenzae in a patient with common variable immunodeficiency (CVID): a case report
}

\author{
CT Hapuarachchi ${ }^{1}$, GK Karunaratne ${ }^{2}$, NR de Silva ${ }^{3}$, EM Corea ${ }^{4}$ \\ Sri Lankan Journal of Infectious Diseases 2018 Vol.8 (1):46-50 \\ DOI: http://dx.doi.org/10.4038/sljid.v8i1.8159
}

\begin{abstract}
Fluoroquinolones are important in the treatment of respiratory infections and resistance to them amongst Haemophilus influenzae is rare. We report a case of persistent respiratory tract colonization with an $H$. influenzae strain resistant to ciprofloxacin, levofloxacin and moxifloxacin.

Two sputum cultures obtained 4.5 months apart, from a patient with common variable immunodeficiency (CVID), yielded $H$. influenzae in colony counts exceeding $10^{7} \mathrm{CFU} / \mathrm{ml}$. Antibiotic susceptibility testing using disk diffusion and E-tests revealed both isolates to be resistant to ciprofloxacin, levofloxacin and moxifloxacin.

Although quinolone resistant $H$. influenzae remains extremely rare, its emergence in Sri Lanka heralds microbiological and clinical challenges with important implications for the treatment of community acquired respiratory infection.
\end{abstract}

Keywords: Fluoroquinolone resistance, Haemophilus influenzae, Sri Lanka

\section{Introduction}

Haemophilus influenzae is an important cause of community acquired respiratory tract infection. Published data for the role of $H$. influenzae in respiratory tract infections in Sri Lanka is not available. The incidence of $H$. influenzae meningitis in Sri Lanka was reported as being20.1 cases per 100000 in 2004. ${ }^{1} \mathrm{H}$. influenzae is the commonest bacterium causing exacerbations in patients with common variable immunodeficiency (CVID). ${ }^{2}$ These patients may be persistently colonized with $H$. influenzae and suffer recurrent respiratory tract infections which in turn may lead to bronchiectasis and increased patient morbidity and mortality.

${ }^{1}$ Department of Microbiology and Infection Control, National Hospital of Sri Lanka, Sri Lanka.

${ }^{2}$ Lady Ridgeway Hospital, Colombo 8, Sri Lanka

${ }^{3}$ Medical Research Institute, Colombo 8, Sri Lanka

${ }^{4}$ Faculty of Medicine, University of Colombo, Sri Lanka

Address for correspondence: Dr CT Hapuarachchi, No. 6/4, St. Sylvester Road, Mount Lavinia, Western Province, Sri Lanka. Telephone number: +94764679898 Email: <thamarahapu@hotmail.com>

(i) http://orcid.org/0000-0002-4166-3922

Received 30 October 2017 and accepted 10 March 2018 
Fluoroquinolones are important antibiotics for treating respiratory infections. Although fluoroquinolone resistant $H$. influenzae isolates have been reported sporadically, they remain extremely rare, with international surveillance data showing 0-0.5\% resistance rates. ${ }^{3,4}$ An extensive literature survey did not reveal any reports of such isolates from Sri Lanka.

\section{Case Report}

A 41 year old lady had been diagnosed with CVID one year previously. She had reduced serum immunoglobulin levels ( $\operatorname{IgG}, \operatorname{IgA}$ and $\operatorname{IgM}$ ), impaired antibody responses to vaccines and absent isohaemagglutinins. Her B lymphocyte count was 368/ $\mu$ l (within normal limits for her age). The patient was started on $25 \mathrm{~g}$ of intravenous immunoglobulins every four weeks.

In the previous seven years she had experienced multiple lower respiratory tract infections necessitating repeated hospital admissions and intravenous antibiotics, including one episode of left side basal pneumonia. At the time of diagnosis she had chronic sputum production with CT scan evidence of central bronchiectasis.

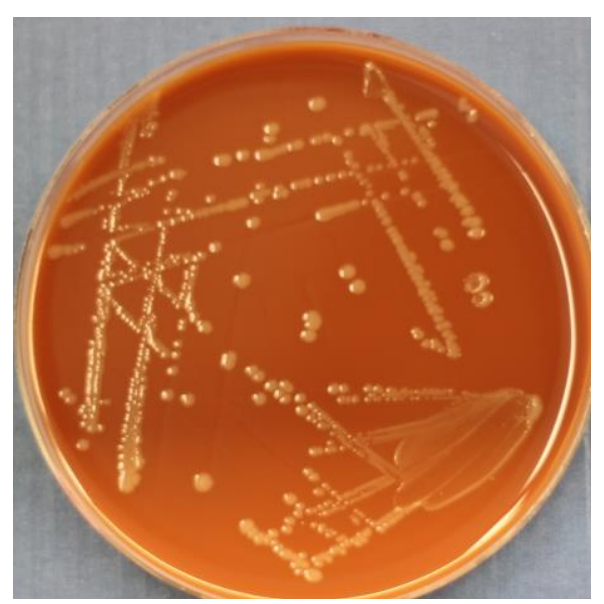

Figure 1: Isolated $H$. influenzae

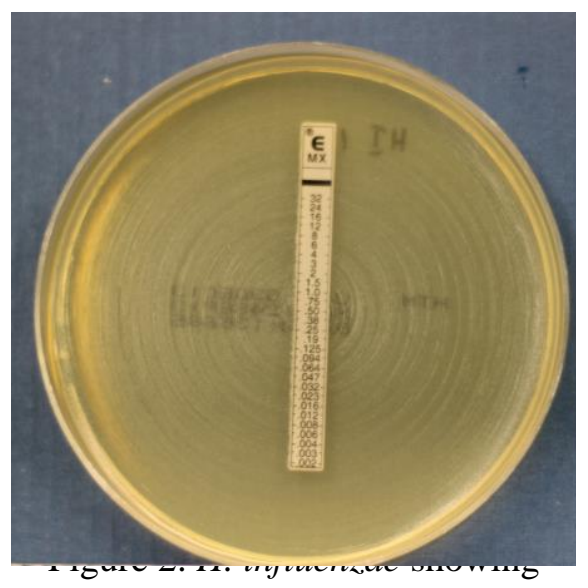

moxifloxacin resistance on Haemophilus Test Medium (MIC $>32 \mu \mathrm{g} / \mathrm{ml}$ )
Quantitative culture of two sputum specimens, four and a half months apart, taken to assess colonizing respiratory flora, yielded $H$. influenzae with colony counts exceeding $10^{7}$ colony forming units/ml (Figure 1). Both were good quality sputa belonging to Murray and Washington's group $5 .^{5}$ During this period the patient was not on any antibiotics and was free of respiratory tract infections or exacerbations.

H. influenzae was identified by its typical Gram stain appearance (poorly staining Gram negative coccobacilli), characteristic 'seminal' odour of colonies on chocolate agar, satellitism around Staphylococcus aureus and requirement for Factor $\mathrm{X}$ and $\mathrm{V}$. Identification was confirmed by matrix-assisted laser desorption and ionization-time of flight mass spectrometry (MALDI-TOF).

Antibiotic susceptibility testing was done by disk diffusion using Haemophilus Test Medium (Oxoid) according to CLSI recommendations and minimum inhibitory concentrations (MICs) were determined by the E-test (bioMérieux). ${ }^{6}$ Beta-lactamase testing was done using both acidometric and biological methods.

The antibiotic susceptibility profiles of both isolates were similar. On disk diffusion testing they were sensitive to ampicillin, amoxicillin-clavulanic acid, cefuroxime, 
ceftriaxone, azithromycin, tetracycline, chloramphenicol and meropenem and resistant to ciprofloxacin, levofloxacin and trimethoprim-sulfamethoxazole. The isolates were negative for beta-lactamase production. E-test confirmed both isolates to be sensitive to ampicillin and ceftriaxone (MICs were 0.19 and $0.08 \mu \mathrm{g} / \mathrm{ml}$ respectively). However, the MIC for moxifloxacin by the E-test was $>32 \mu \mathrm{g} / \mathrm{ml}$ (Figure 2), indicating high level moxifloxacin resistance (defined as an MIC $>32 \mu \mathrm{g} / \mathrm{ml}$ ). Between September 2012 and April 2013, 28 patients with primary antibody deficiency attending the Immunology Clinic at the MRI were screened by asking them to submit two samples taken 4.5 months apart. Sputum was collected from chronic sputum producers and oral rinses from others and were quantitatively cultured for possible respiratory pathogens. A similarly resistant $H$. influenzae was not isolated from any samples collected during this period. ${ }^{7}$

Although this patient was on regular immunoglobulin replacement therapy since diagnosis and during the period of sputum examinations, her trough IgG levels remained inadequate. Her last documented trough IgG was $422 \mathrm{mg} / \mathrm{dl}$ (ref level for age and sex 569-1919 mg/dl) which was taken one month before the second sputum sample. As her trough immunoglobulin level was inadequate with persistent symptoms, the immunoglobulin dose was increased to $30 \mathrm{~g}$. However, two months after her immunoglobulin dose was increased, she developed fever, chills, rigors and wheezing to IVIG. A closely supervised re-exposure elicited similar symptoms accompanied by hypotension. IVIG was stopped and as subcutaneous immunoglobulin replacement therapy is not available in Sri Lanka, replaced with IVIG given subcutaneously. She developed similar symptoms. She has therefore not been on any replacement therapy for the past 5 years. Although she has frequent clinical respiratory exacerbations she continues her routine daily activities.

\section{Discussion:}

Ciprofloxacin resistant $H$. influenzae was first reported over two decades ago in a patient with severe combined immunodeficiency. ${ }^{3}$ Since then, this resistance phenotype has been reported sporadically and has remained rare. Mutations in the quinolone resistance determining region of the topoisomerase II and I remain the commonest mechanism of fluoroquinolone resistance and this is likely to be the probable mechanism in these isolates as well. ${ }^{3}$ Quinolone resistance is associated with previous quinolone therapy. ${ }^{3}$ There was no documented evidence of preceding fluoroquinolone therapy in our patient. However, she had a history of multiple lower respiratory infections which had been treated by oral and parenteral antibiotics, although relevant details were unavailable. Given the widespread empirical use of quinolones in lower respiratory tract infections by medical practitioners in Sri Lanka, it is quite likely that a past exposure was possible.

Several clonal outbreaks of quinolone resistant $H$. influenzae have been documented, illustrating the potential of these isolates for dissemination. ${ }^{8}$ The emergence of such strains in Sri Lanka has implications for infection control, given the fact that patients are managed at crowded immunology and chest disease clinics where many susceptible patients are likely to congregate.

Our patient is a housewife and her family and household contacts are healthy without any chest pathology. She had regularly attended the immunology and respiratory outpatient clinics, been to several general practitioners and been hospitalized three times for respiratory tract infections. 
Unfortunately, given the existing resources and surveillance systems currently in place, it is therefore not possible to determine the possible source of her colonizing fluoroquinolone resistant $H$. influenzae.

Cohort segregation of cystic fibrosis (CF) patients with Burkholderia cepacia complex (and within the complex) is widely practiced in CF units worldwide. Some "epidemic" multi resistant $P$. aeruginosa strains have also been brought under control through strict segregation and infection control practices. ${ }^{9}$ However there is no conclusive evidence that such methods would have the same impact in bronchiectatic patients without $\mathrm{CF}$, who are the predominant case mix in Sri Lanka. Better understanding of the colonizing flora of such patients and their relationship to patient morbidity may be needed before extrapolating the same approaches used in $\mathrm{CF}$ units. Furthermore, segregation with strict infection control practices of these patient groups would be suboptimal without continuous, prospective surveillance of respiratory pathogens and sensitive typing methods.

\section{Conclusion}

Even though quinolone resistant $H$. influenzae remains extremely rare, its emergence in Sri Lanka presents microbiological and clinical challenges with important implications for the treatment of community acquired respiratory infections. Judicious use of fluoroquinolones in the treatment of patients with chronic lung diseases to prevent emergence of resistance, improving laboratory capability to detect resistant strains and timely infection control measures to limit cross infection will mitigate the danger posed by these organisms.

\section{Acknowledgements}

None

\section{Conflicts of Interest}

There are no conflicts of interest.

\section{References}

1. Batuwanthudawe R, Rajapakse L, Somaratne P, et al. Incidence of childhood Haemophilus influenzae type b meningitis in Sri Lanka. Int J Infect Dis 2010; 14: e372-6.

doi: http://dx.doi.org/10.1016/j.ijid.2009.06.018

2. Samuelson, A, Borrelli S, Gustafson R, et al. Characterization of Haemophilus influenzae isolates from the respiratory tract of patients with primary antibody deficiencies: evidence for persistent colonizations. Scand. J. Infect. Dis. 1995; 27:303-313.

doi: http://dx.doi.org/10.3109/00365549509032722

3. Barriere SL, Hindler JA. Ciprofloxacin resistant Haemophilus influenzae infection in a patient with chronic lung disease. Ann Pharmacother 1993; 27:309-310.

doi: http://dx.doi.org/10.1177/106002809302700311

4. Pfaller MA, Farrell DJ, Sader HS, et al. AWARE Ceftaroline Surveillance Program (2008-2010): trends in resistance patterns among Streptococcus pneumoniae, Haemophilus influenzae, and Moraxella catarrhalis in the United States. Clin Infect Dis 2012; 55: S187-93.

doi: http://dx.doi.org/10.1093/cid/cis561 
5. Murray PR, Washington JA. Microscopic and bacteriologic analysis of expectorated sputum. Mayo Clin Proc 1975; 50:(6):339-344. No doi

6. Clinical and Laboratory Standards Institute. Performance Standards for Antimicrobial Susceptibility Testing. 21st informational supplement. M100-S22, Clinical and Laboratory Standards Institute, 2012.

7. Hapuarachchi CT, de Silva NR, Corea EM, et al. Respiratory colonizers in patients with primary antibody deficiencies attending an immunology clinic in Sri Lanka. The Bulletin of the Sri Lanka College of Microbiologists 2015; 13 (1): 13. No doi

8. Nazir J, Urban C, Mariano N, et al. Quinolone-resistant Haemophilus influenzae in a long-term care facility: clinical and molecular epidemiology. Clin Infect Dis. 2004; 38:1564-9.

doi: http://dx.doi.org/10.1086/420820

9. Griffiths AL, Jamsen K, Carlin JB, et al. Effects of segregation on an epidemic Pseudomonas aeruginosa strain in a cystic fibrosis clinic. Am J Respir Crit Care Med 2005; 171:1020-1025 doi: http://dx.doi.org/10.1164/rccm.200409-1194OC 